\title{
Descripción de política pública de primera infancia en el Instituto Colombiano De Bienestar Familiar - Cereté.
}

\author{
Description of public policy of early childhood in the Colombian Institute Of \\ Family Welfare - Cerete.
}

\author{
Dilia Cristina Chávez Almario, Mónica Hanna Lavalle \\ Administradora en Salud, Universidad de Córdoba. \\ diliacchavez@hotmail.com
}

\begin{abstract}
RESUMEN
Introducción: describir la Política Pública de Primera Infancia del Instituto Colombiano de Bienestar Familiar del municipio de Cerete, 2015. Métodos: descriptiva, retrospectiva, enfoque cuantitativo. Población 3.819 personas, muestreo aleatorio simple y muestra 349 usuarios. Se realizó una encuesta estructurada, con trece (13) ítems. Resultados: el 60,5\% y el 65,5\% de los usuarios de los Hogares Comunitarios de Bienestar Tradicional y Hogares Fami, manifestaron que el mobiliario es inadecuado, a diferencia de centros de desarrollo infantil en los cuales el $88,2 \%$ de usuarios contaban con mobiliario adecuado y seguro. Así mismo el $69,7 \%$ y el $62,7 \%$ de los Hogares Comunitarios de Bienestar Tradicional y Fami manifestaron no contar con buenas condiciones de aseo y limpieza, el $85,2 \%$ de usuarios de los centros de desarrollo infantil afirmaron lo contrario. En cuanto al desarrollo de capacidades motrices y cognitivas, los padres afirmaron que los niños(as) beneficiarios de los programas alcanzaron buen desarrollo $85,8 \%$ y $82,8 \%$ en estas áreas desde que asisten a estos centros. La morbilidad sentida fue Enfermedad Diarreica Aguda 70\%, Infección Respiratoria Aguda 60,5\%. La confianza que proyectan los coordinadores de los programas a los padres tuvo una media de $48,8 \%$. Conclusiones: la Política Pública de Primera Infancia del instituto, posee fortalezas en los centros de desarrollo infantil y se percibió debilidad en otros programas con relación a estándares establecidos por normatividad vigente.
\end{abstract}

Palabras Clave: Política Pública, Infancia, Evaluación, Métodos, Ética.

\section{ABSTRACT}

Introduction: to describe the public policy of first children of the Colombian Institute of family welfare of the municipality of Cereté, 2015. Methods: descriptive, retrospective, quantitative approach. Population 3.819 people, simple random sampling and shows 349 users. A structured survey, with thirteen (13) items was conducted. . Results: 60.5 per cent and $65.5 \%$ of the users 
of traditional welfare and household Fami community homes, hand furniture is communicate, unlike child development centers in which $88.2 \%$ of users were safe and proper furniture. Likewise $69.7 \%$ and $62.7 \%$ of the community homes of traditional welfare and Fami manifest not having good conditions of cleaning, $85.2 \%$ of users of child development centers stated otherwise. In terms of the development of motor and cognitive skills, parents expressed that the children beneficiaries of programs achieved good development $85.8 \%$ and $82.8 \%$ in these areas since attending these centers. Silymarin acute Diarrhea $70 \%$, acute respiratory infection, felt morbidity was 60.5 per cent. Confidence projected by the programs coordinators the parents had an average of $48.8 \%$. Conclusions: Early childhood Institute of public policy, has strengths in the child development centers and was perceived weakness in other programs in relation to standards set by current regulations.

Keywords: Public politics, childhood, evaluation, method, ethics.

\section{INTRODUCCIÓN}

La Constitución Política de Colombia señala que la vida, la integridad física y la salud, entre otros, son derechos fundamentales de los niños y niñas. Gozar de condiciones para su desarrollo es requisito esencial para que puedan aprender, desarrollar sus capacidades, adquiriendo aptitudes necesarias para una vida en sociedad.

Es así que desde la primera infancia, se construyen las condiciones para el ingreso del niño y de la niña a la vida social y cultural. Es en la primera infancia donde se busca un desarrollo integral de los menores a través de acciones saludables, como lo son la nutrición adecuada, ambientes favorables, la escucha y participación activa de los mismos permitiendo construir su identidad, promocionado o restablecidos así sus derechos fundamentales.

En este sentido la Política de Primera Infancia incorpora la perspectiva de la protección integral, la cual, entre otros aspectos, proporciona un marco para analizar la situación de los niños y las niñas, los pone en la condición de sujeto titular de derechos, establece el interés superior del niño y de la niña como el criterio o parámetro fundamental, para tomar decisiones, considera que la familia, la sociedad y el Estado son corresponsables del cumplimiento de dichos derechos (1).

La movilización por la primera infancia a nivel nacional tuvo origen en el 2002, en la Alianza por la Política Pública de Infancia y Adolescencia en Colombia, a través de un grupo de trabajo integrado por el Instituto Colombiano de Bienestar Familiar ICBF, el Departamento Administrativo de Bienestar Social DABS, el Centro Internacional de Educación y Desarrollo -CINDE-, Save the Children y UNICEF. En el 2004 este grupo de trabajo se amplía a 19 instituciones bajo la coordinación del ICBF (2).

Siendo que el Instituto Colombiano de Bienestar Familiar es el escenario donde se materializa la Política Publica de Primera Infancia, a través de la ejecución de sus programas, debe utilizar metodologías que permitan medir que tan efectivos han sido, si los objetivos se han alcanzado y si han logrado impactos positivo en sus beneficiarios. 
Es por ello, que esta investigación describió la Política Pública de Primera infancia en el Instituto Colombiano de Bienestar Familiar en Cerete (Córdoba. Colombia) en el año 2015, materializando los programas Centros de Desarrollo Infantil (CDI), Hogares Comunitarios de Bienestar HCB FAMI y Tradicional para indagar sobre su efectividad y así poder proponer lineamientos que fortalezcan la política, garantizando un elevado porcentaje el bienestar de los futuros adultos del país.

\section{MÉTODOS}

Se realizó un estudio descriptivo, retrospectivo, con enfoque cuantitativo. La población estuvo constituida por niños y niñas de cero (0) meses a 5 años de edad y mujeres gestantes y lactantes que fueron beneficiarias de los programas del Instituto Colombiano de Bienestar Familiar del municipio de Cereté que están vinculada a los Centros de Desarrollo infantil (CDI), hogares comunitarios de bienestar tradicional (HCBT) y hogares comunitarios de bienestar FAMI (HCBF) que de manera agrupada suman 3.819 usuarios. Para determinar el tamaño de la muestra, se aplicó la fórmula estadística para estimación de una proporción para poblaciones finitas que arrojo 349 unidades. El tipo de muestreo utilizado fue aleatorio simple (3), eligiendo al azar a los objetos de estudio. Se visitaron todos los Centro de Desarrollo Infantil (CDI), Hogares Comunitarios Tradicionales (HCBT) y Hogares Comunitarios FAMI (HCBF) y aleatoriamente se seleccionaron padres de familia, mujeres gestantes y lactantes, a los cuales se les realizaron las encuestas diseñadas para cada programa, se les explico en qué consistía el estudio y se confirmó la aceptación a participar a través del consentimiento informado, posteriormente se dispusieron a realizar el llenado de la encuesta. Para la recolección y tratamiento de los datos se aplicó una encuesta tipo escala Likert que incluyen los ítems necesarios para el estudio, con 13 preguntas que permitieron obtener la información requerida y de manera confidencial.

Para la recolección de los datos se dividieron proporcionalmente las 349 encuestas entre los beneficiarios de los programas, es decir, el total de los niños y niñas, las gestantes y lactantes, lo cual permitió establecer el número de encuestas a aplicar así: niños(as) de los centros de desarrollo infantil (CDI) 98, de los hogares comunitarios de bienestar FAMI 63 y hogares comunitarios de bienestar TRADI 116; Gestantes de los FAMI 19 Y TRADI 16; lactantes de los FAMI 17 y TRADI 20.

Tabla 1. Distribución proporcional de la muestra.

\begin{tabular}{|c|c|c|c|}
\hline Programa & Padres & Gestantes & Lactantes \\
\hline Centros de Desarrollo Infantil (CDI) & 98 & 0 & 0 \\
\hline $\begin{array}{l}\text { Hogares Comunitarios de Bienestar } 63 \\
\text { FAMI }\end{array}$ & & 19 & 17 \\
\hline $\begin{array}{l}\text { Hogares Comunitarios de Bienestar } 11 \\
\text { Tradicional }\end{array}$ & & 16 & 20 \\
\hline
\end{tabular}

Para la validez del instrumento se sometió al juicio de cinco (5) expertos, los cuales analizaron sistemáticamente, así como la estructura del instrumento. Estos expertos emitieron sus juicios y consideraciones respecto a la redacción y contenido, verificando 
si los ítems median las variables y sus respectivos indicadores; así como los objetivos de la investigación.

Para estimar la confiabilidad de los ítems, se aplicará una prueba piloto en un número de sujetos en condiciones similares a las investigadas, esta actividad fue realizada en un hogar de bienestar del municipio de Montería, el cuál reunía las condiciones y características similares de la población objeto de estudio. Se aplicó un total de 40 encuestas para detectar dificultades en las preguntas formuladas, una vez recopilada la información se aplicó la fórmula de confiabilidad Alfa de Cronbach, utilizando para ello el siguiente teorema estadístico:

$$
r_{t t}=\frac{K}{K-1}\left[1-\frac{\sum S_{i}^{2}}{S_{t}^{2}}\right]
$$

Dónde:

$\mathrm{rtt}=$

$\mathrm{K}=$

$\mathrm{Si}=\quad$ varianza de los puntajes de cada ítems $\mathrm{St}=$

coeficiente de confiabilidad número de ítems varianza de los totales.

Tomando en cuenta la fórmula expresada, se calculó el coeficiente para la confiabilidad de instrumentos con ítems continuos los cuales fueron aplicados para los sujetos de la investigación, arrojo un Alpha de 0.96, determinando la confiabilidad del instrumento y validez del mismo.

De alguna manera son un indicador de relación global entre los ítems, aunque no equivalen a la correlación media entre los ítems, por esta razón después de la construcción y análisis estadístico para cada uno de los instrumentos se indican las variables que poseen confiabilidad con respecto al instrumento, al ser comparadas con la escala de valoración que se presenta a continuación, la cual es herramienta estadísticas para determinar la validez de variable.

Tabla 2. Escala para la valoración del coeficiente de confiabilidad.

\begin{tabular}{|l|l|}
\hline RANGOS & MAGNITUD \\
\hline 0,81 a 1,00 & Muy alta \\
\hline 0,61 a 0,80 & Alta \\
\hline 0,41 a 0,60 & Moderada \\
\hline 0,21 a 0,40 & Baja \\
\hline 0,01 a 0,20 & Muy baja \\
\hline
\end{tabular}

Fuente: Ruiz (2002)

Para el análisis de los datos después de procesados en el programa estadístico SPSS versión 21 , se cuantificaron en función de las frecuencias y porcentajes de los datos recolectados.

Además de lo anterior, se tuvieron en cuenta los estándares de calidad del anexo técnico para orientar la prestación de servicios en centros de desarrollo infantil y los lineamiento técnico, administrativo y operativo, modalidad hogares comunitarios de bienestar en todas sus formas (fami, familiares, grupales, múltiples, múltiples empresariales y jardines 
sociales) para la atención a niños y niñas hasta los cinco (5) años de edad del Instituto Colombiano de Bienestar Familiar.

\section{RESULTADOS}

Caracterización de la Población Beneficiaria y Modalidades de Prestación del Servicio En total, el Instituto Colombiano de Bienestar Infantil atendió en el Municipio de Cerete para la vigencia 2015 en sus diferentes modalidades a 3.819 beneficiarios así: niños y niñas. 3.029, Mujeres Gestantes 390 y mujeres Lactantes 400.

\section{Modalidad integral}

La modalidad integral la conforman los Centros de Desarrollo Infantil (CDI), para el año 2015 en el municipio de Cerete, en las unidades básicas de atención UBA, la población estuvo constituida en un $70 \%$ por niños con edad menor o igual a 5 años y el $30 \%$ mujeres gestantes y lactantes, se pudo constatar que estos Centros de Atención Integral, fueron infraestructuras construidas con recursos CONPES y del Instituto Colombiano de Bienestar Familiar, o una combinación de ambos. La tabla 3 resume la información relacionada con la población beneficiada de esta modalidad.

Cada Unidad Básica de Atención, contaba con un equipo interdisciplinario, los cuales aglutinaban profesionales de las siguientes áreas: Nutrición y Dietética, Trabajo social, y Pedagogía.

Tabla 3. Población Beneficiada Centros de Desarrollo Infantil en Cerete, año 2015.

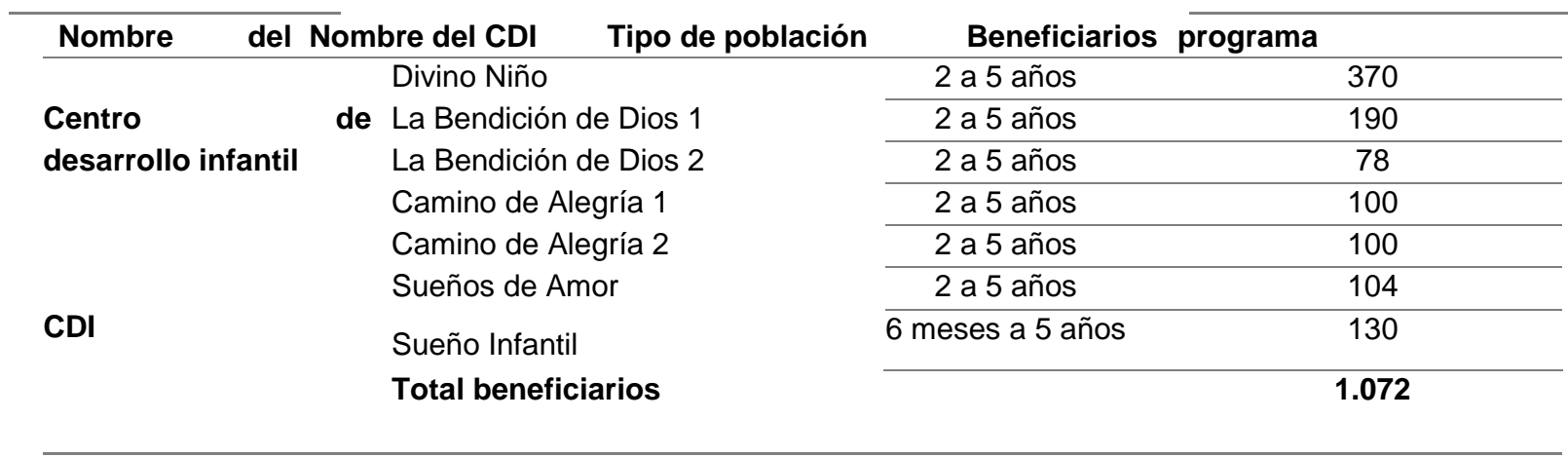

Fuente: Instituto Colombiano de Bienestar Familiar sede Cerete.

Como se muestra en la tabla 3, la población infantil beneficiaria fue 1072, siendo el $48 \%$ niños y niñas el $52 \%$.

\section{Modalidad no integral}

En la modalidad no integral destacan dos categorías: los hogares comunitarios de bienestar tradicional (HCB-Tradicional), y los hogares comunitarios de bienestar familia mujer e infancia (HCB- FAMI), en esta modalidad se pudo observar que la mayor parte de la población venia de condiciones de vulnerabilidad. Las tablas 4 y 5 , describen la población beneficiaria en estas categorías. 
Tabla 4. Hogares Comunitarios de Bienestar FAMI

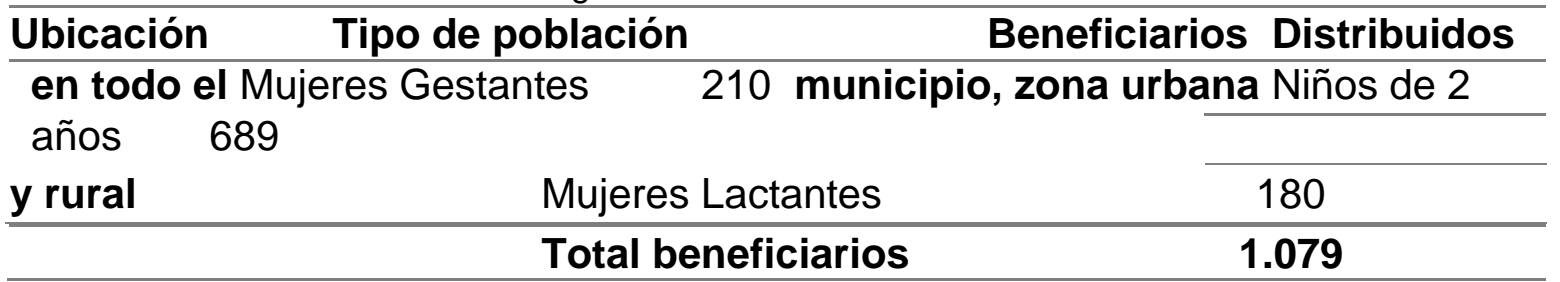

Fuente: Instituto Colombiano de Bienestar Familiar sede Cerete.

Como se observa en la tabla 4 la población beneficiaria de la categoría FAMI, fue de 1079 , de los cuales el 30\% fueron niños; $34 \%$ niñas; $19 \%$ mujeres gestantes, y mujeres lactantes $17 \%$.

Tabla 5. Hogares Comunitarios de Bienestar Tradicional.

\begin{tabular}{|c|c|c|}
\hline \multirow[t]{2}{*}{ Ubicación } & Tipo de Población & Beneficiarios \\
\hline & Mujeres Gestantes & 180 \\
\hline \multicolumn{2}{|c|}{$\begin{array}{l}\text { Distribuidos en todo el } \\
\text { municipio, zona urbana Niños de } 2 \text { a } 5 \text { años }\end{array}$} & 1.268 \\
\hline y rural & Mujeres Lactantes & 220 \\
\hline & Total beneficiarios & 1.668 \\
\hline
\end{tabular}

Fuente: Instituto Colombiano de Bienestar Familiar sede Cerete.

La tabla anterior muestra que para la categoría HCB-Tradicional, fueron beneficiados 1668 , de esta población, el 11\% fueron mujeres gestantes; $13 \%$ mujeres lactantes, y el $76 \%$ niños de 2 a 5 años.

\section{Condiciones de infraestructura para prestación del servicio}

Se pudo observar que los beneficiarios de los Hogares Comunitarios de Bienestar Tradicionales (HCB-Tradicional) y los Hogares Comunitarios de Bienestar FAMI (HCBF) no contaban con mobiliario adecuado y seguro para los menores, a diferencia de los Centros de Desarrollo Infantil (CDI) en los cuales el $88,24 \%$ de los usuarios manifesto que estos se encontraban en buenas condiciones, estos resultados se observan en el grafico 1.

Grafico 1. Distribución del mobiliario de acuerdo a la condición adecuado y seguro. 


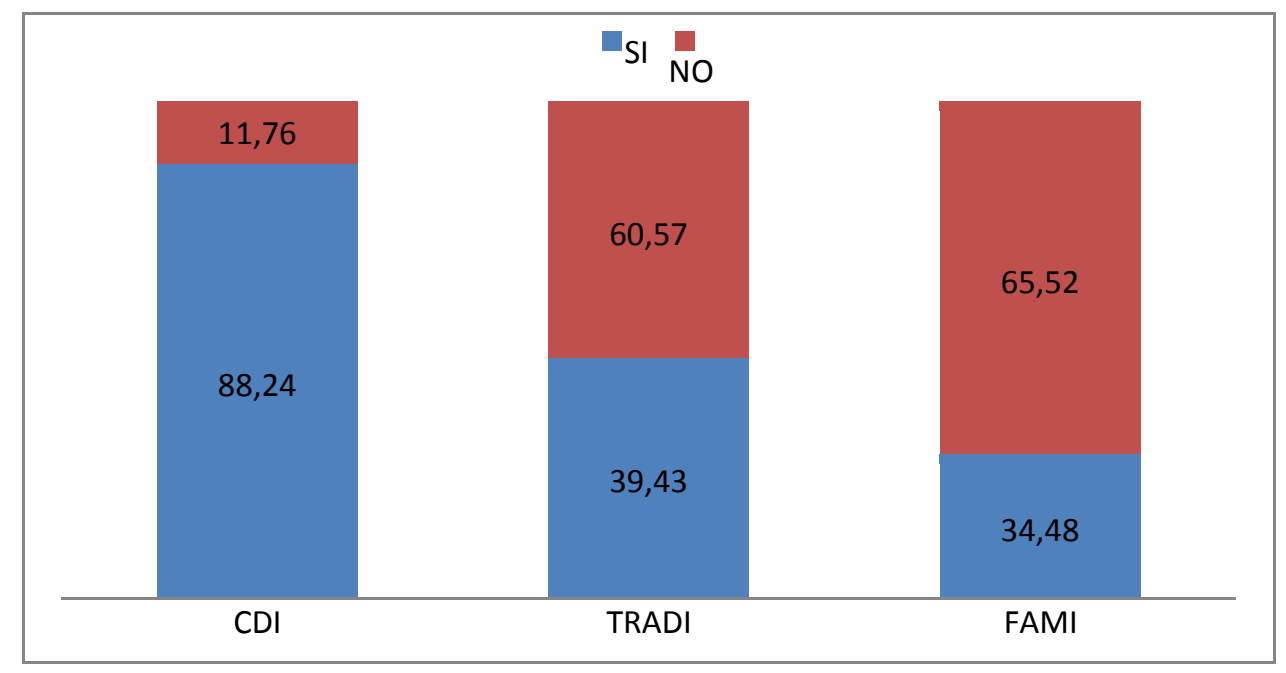

Fuente: Elaboración propia - Instituto Colombiano de Bienestar Familiar sede Cerete, 2015.

Al comparar los tres programas como lo muestra el grafico anterior, en relación al mobiliario un alto porcentaje de los encuestados manifestaron que los Centros de Desarrollo Infantil CDI a diferencia de los Hogares Comunitarios de Bienestar Tradicional y Hogares Comunitarios de Bienestar FAMI, cuentan con instalaciones dotadas de mobiliario adecuado y seguro lo que favorece un ambiente propicio para el desarrollo de las actividades lúdicas y recreativas de los menores.

En cuanto a las condiciones de aseo y limpieza, el $85,2 \%$ de los usuarios de los CDI, manifiesto que son buenas, a diferencia de los HCB Tradicionales y FAMI en los cuales el $69,7 \%$ y $62,7 \%$ de los usuarios manifesto que estos centros no contaban con buenas condiciones de aseo y limpieza. Estos resultados se resumen en el grafico 2.

Grafico 2. Distribución de la opinión de acuerdo a la condición aseo y limpieza

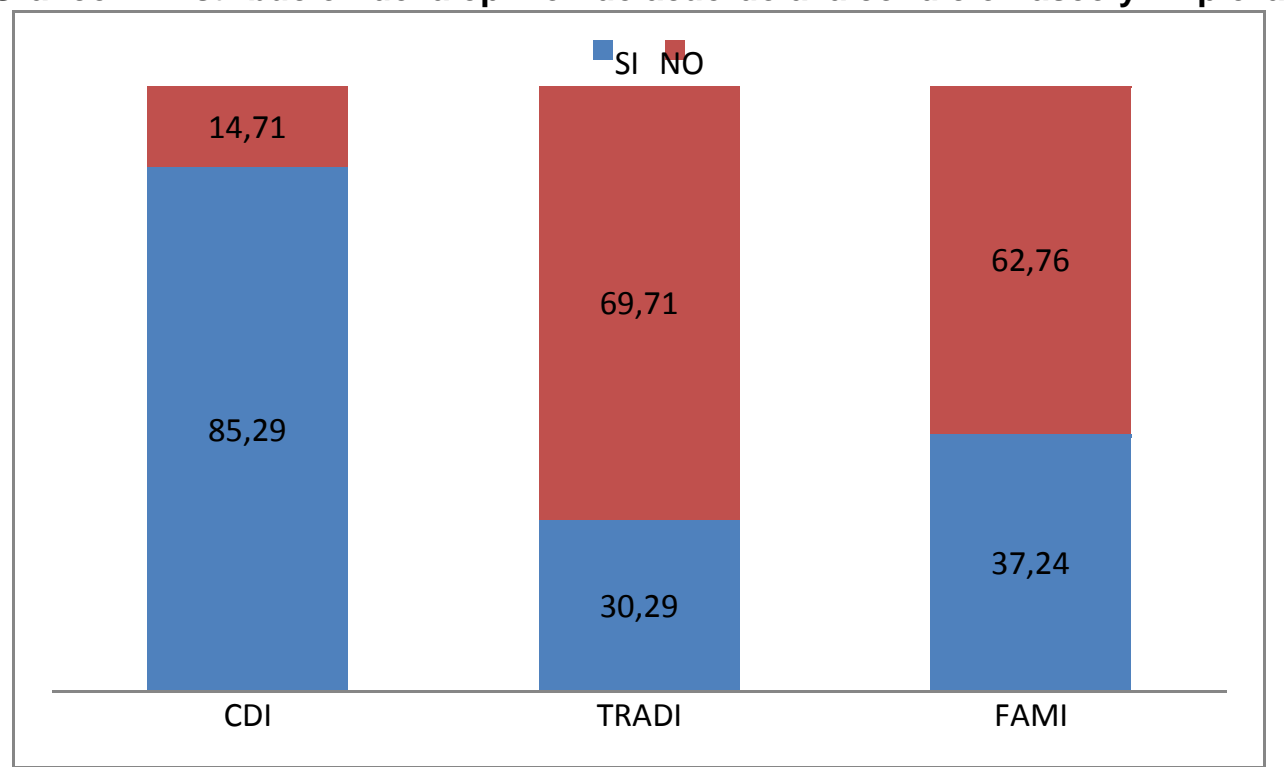

Fuente: Elaboración propia - Instituto Colombiano de Bienestar Familiar sede Cerete, 2015. 
El grafico 3 muestra la morbilidad sentida en los niños del estudio, se observa que la enfermedad que mas se manifesto en los CDI y en los HCB tradiconal es IRA, que alcanza un $70 \%$ y $60,57 \%$ respectivamente, en comparacion con la EDA que no supera el $40 \%$.

Grafico 3. Morbilidad sentida

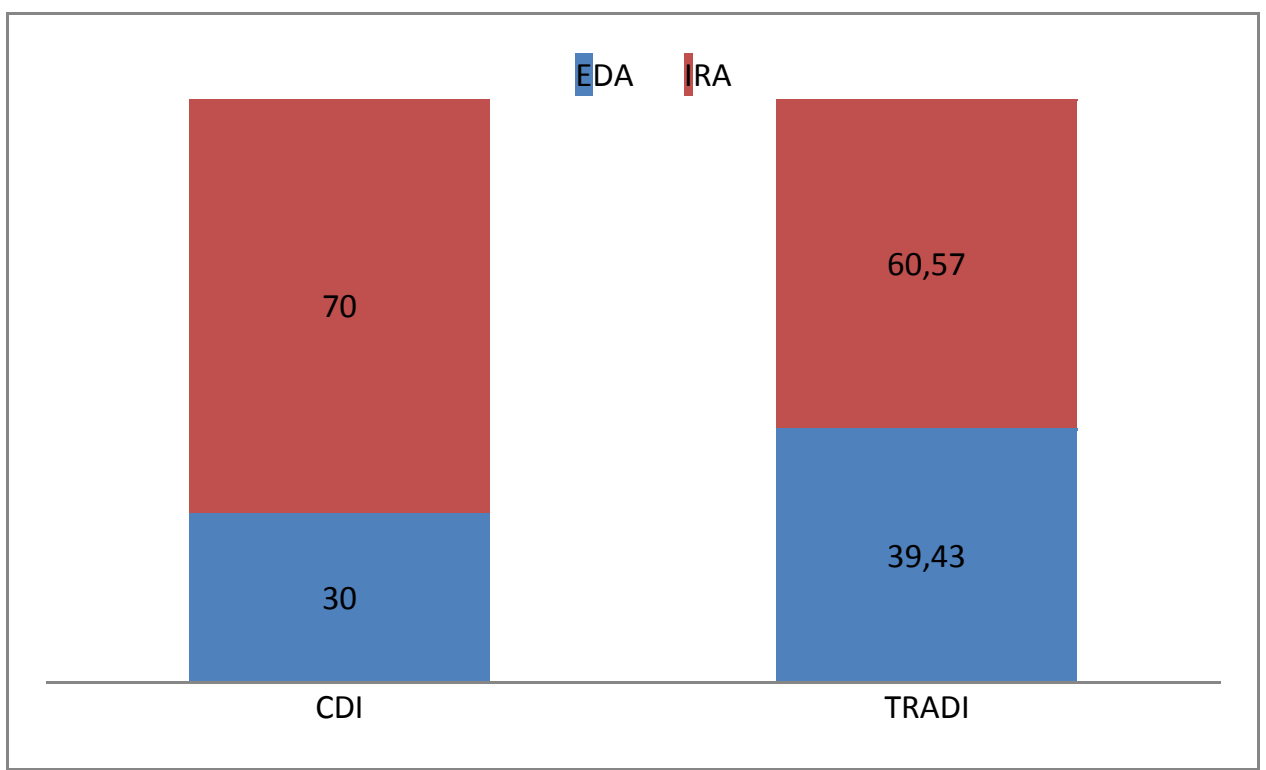

Fuente: Elaboración propia - Instituto Colombiano de Bienestar Familiar sede Cerete, 2015.

\section{Orientacion a los niñso(as) en casa}

Con respecto al apoyo al proceso de formacion, los encuestados de los CDI y los HCB tradicional afirman en su mayoria acompañamiento al proceso $72,94 \%$ y $51,43 \%$, respectivamente para cada servicio. Al comparar los 2 programas se observa que en los CDI el apoyo en casa al proceso de orientacion fue mayor $72,94 \%$, que en los tradicionales $51,43 \%$. El grafico 4 resume estas observaciones.

Grafico 4. Distribución del apoyo al proceso de orientación. 


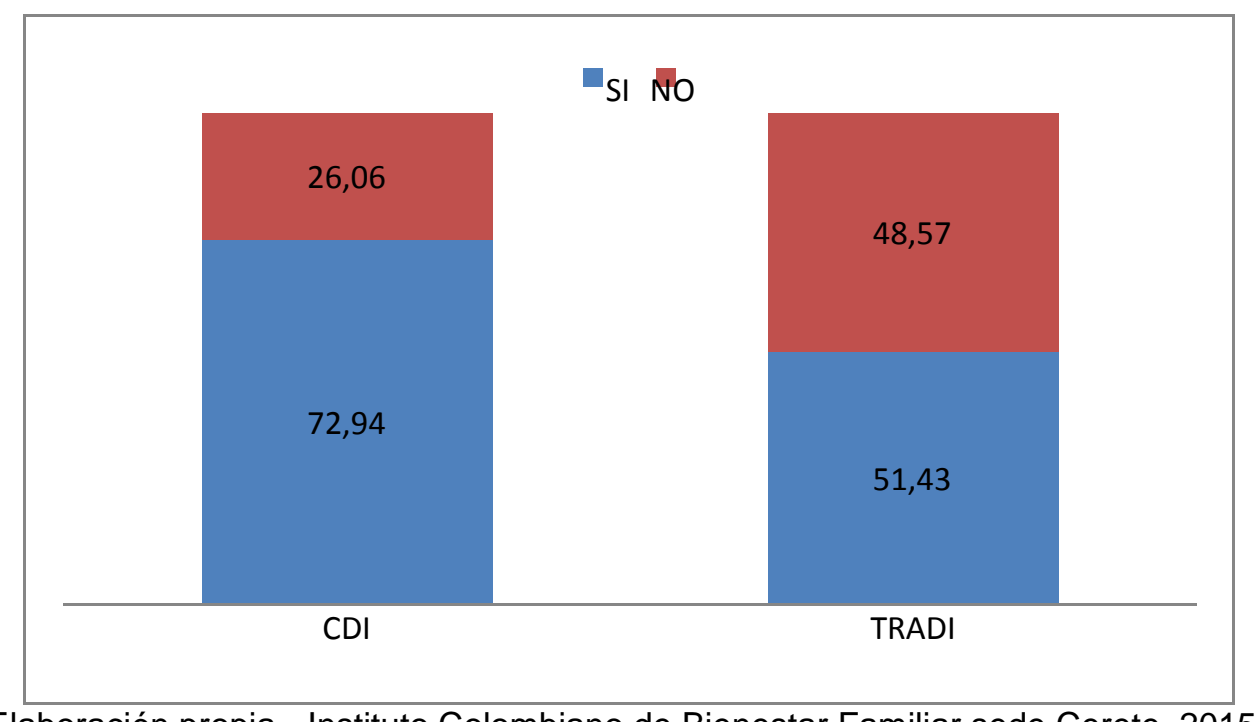

Fuente: Elaboración propia - Instituto Colombiano de Bienestar Familiar sede Cerete, 2015.

\section{Temas para desarrollar en la escuela de padres}

Por otro lado, a la pregunta respecto a los temas en los cuales desean ser capacitadas las beneficiaras de los HCB FAMI, la mayoria respondieron postivamente a Planificacion Familiar con un $45 \%$, y en ese orden Convivencia Familiar alcanza un $25 \%$ de respuestas. Como se observa en el grafico 5 .

Grafico 5. Distribución de los temas de interés para capacitaciones.

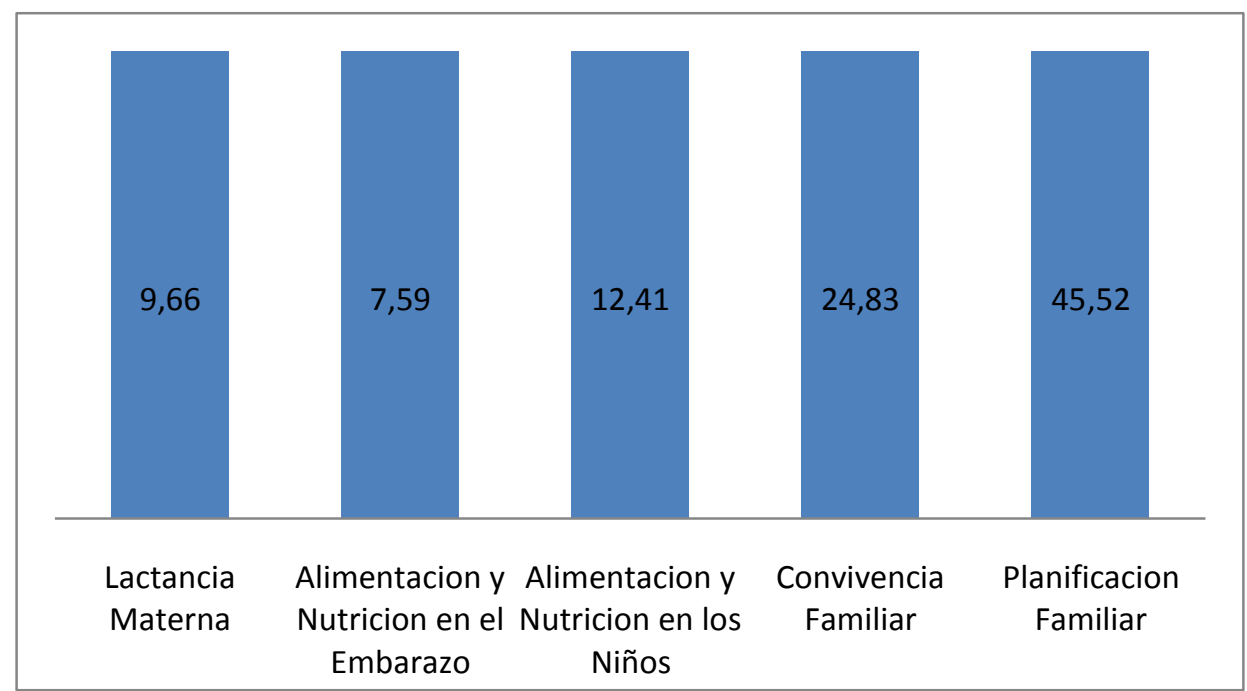

Fuente: Elaboración propia - Instituto Colombiano de Bienestar Familiar sede Cerete, 2015.

\section{Características personales y gestión del personal a cargo de los centros.}

El estudio también permitió indagar sobre las características personales de los encargados de los centros en lo referente a la confianza, interés por solucionar problemas (gestión) y horarios establecidos. En cuanto a la confianza, en porcentajes de 52,9\%. $57,1 \%$ y $36,5 \%$ los usuarios de los centros de desarrollo infantil (CDI) y de los hogares comunitarios de bienestar tradicional y FAMI manifestaron que el equipo interdisciplinario 
de los CDI y las madres comunitarias de los otros centros "algunas veces les inspiran confianza".

Grafico 6. Distribución de la confianza que inspiran los coordinadores entre los usuarios.

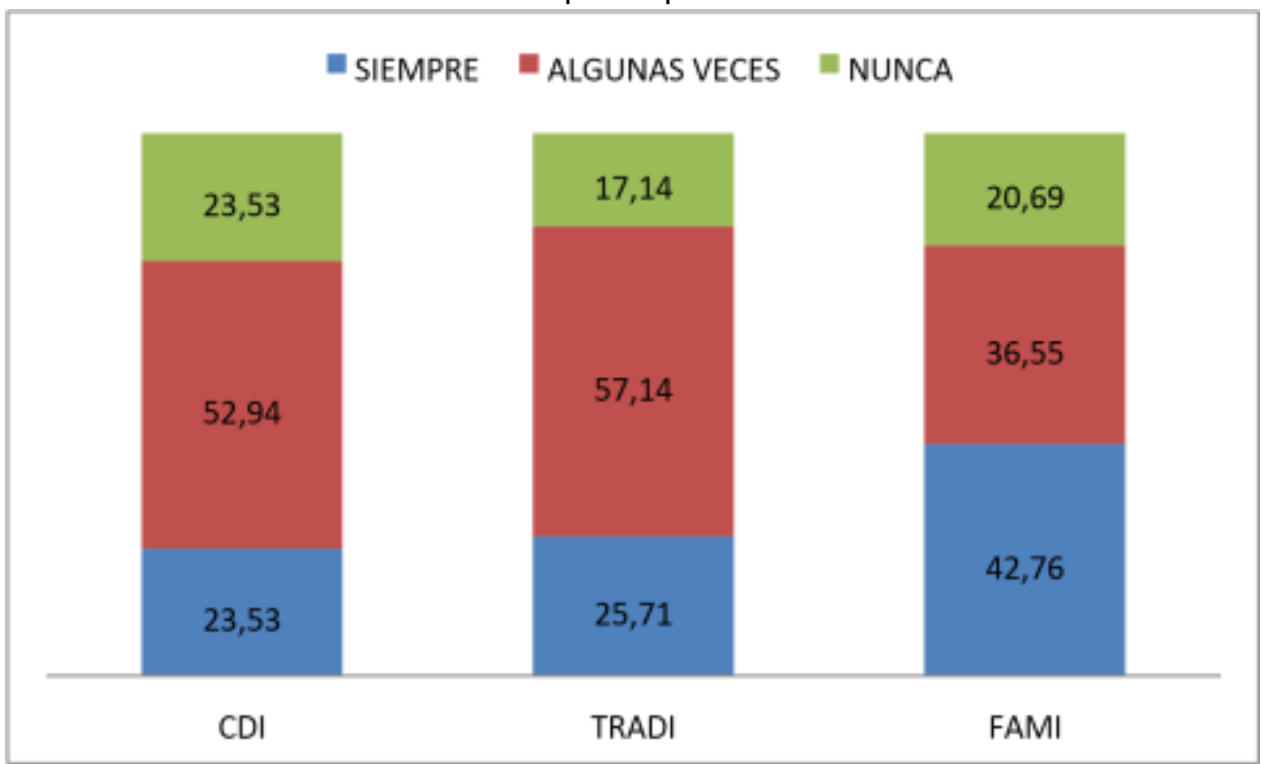

Fuente: Elaboración propia - Instituto Colombiano de Bienestar Familiar sede Cerete, 2015.

\section{DISCUSIÓN}

Los resultados de esta investigación concuerdan con lo descrito en investigaciones realizadas en Leticia (4) en la cual se hallaron debilidades en este tipo de servicios en lo que tiene que ver con la infraestructura y el mobiliario.

Así mismo, las buenas condiciones de aseo y limpieza, no permiten la proliferación de enfermedades transmitidas por vectores y evitan complicaciones en la salud. Lo contrario ocurre en los TRADI y FAMI, donde la falta de mobiliario adecuado y seguro y malas condiciones de limpieza y aseo influyen negativamente en los menores. La inversión gubernamental directa en los CDI influye directamente en su condición, de ahí que estos presenten mejores condiciones de infraestructura, lo contrario ocurre en los otros tipos de centros que básicamente son los casas de las madres comunitarias, que regularmente viven en condiciones de pobreza.

El aseo y la limpieza son condiciones que históricamente han estado relacionadas con los procesos salud- enfermedad, por ello el desarrollo normal de los niños debe tener como condición inexorable un ambiente inocuo, en el cual la capacidad agresora de los microorganismos y su cantidad haya sido diezmada, disminuyendo la morbimortalidad asociada a estos gérmenes, respecto a esto el ICBF (5) plantea que las construcciones para atender a niños deben ser ventilados y es fundamental promover su limpieza y mantenimiento. 
A partir del estudio se determinó que existe un efecto negativo sobre el estado de salud medida con base en la morbilidad sentida de la IRA, sin embargo, este efecto negativo se reduce con la duración de la exposición al programa. La infecciones respiratorias agudas IRA, es la enfermedad que se presentan en mayor proporción lo que afecta la salud de los menores impidiendo su normal asistencia al programa. En muchas ocasiones se han observado focos infecciosos en más del $30 \%$ de los niños y se ha optado por enviarlos a sus casas hasta que mejore su salud como medida preventiva. Estas afecciones pueden ser de origen hídrico como también transmitidas por alimentos como los que se proveen en hogares del ICBF. Las enfermedades respiratorias y las diarreas son las afecciones más frecuentes en la infancia y en mayor medida son de etiología viral y transmitidas por el agua, lo cual concuerda con lo expuesto con Pino et al (6) en su investigación sobre enfermedad diarreica aguda y su relación con la calidad de agua de consumo.

Se destaca del estudio que el acompañamiento de los padres fue fundamental para alcanzar niveles óptimos de desarrollo en los niños y niñas, en este sentido, la investigación nos condujo a pensar que es positivo el acompañamiento de los padres, quienes apoyan en casa las actividades académicas y acatan indicaciones en cuanto a alimentación adecuada, manipulación de los alimentos y en general normas de aseo y limpieza.

El programa Hogares Comunitarios de Bienestar Familiar FAMI, cuyo objetivo principal es orientar a las mujeres gestantes y lactantes acerca de una nutrición adecuada en la gestación y sobre la lactancia materna exclusiva y hasta los 6 meses, además de la protección integral del menor, el estudio determino que estos temas no representan gran interés en la mayoría de beneficiarias. La Planificación y la Convivencia Familiar son los temas en los cuales este grupo de mujeres desean capacitación.

Situación que contrasta con la investigación realizada por Conejeros M, Rojas $\mathrm{J}$ y Segure $\mathrm{T}$ (7) cuyos resultados advierten que la confianza no es considerada como un pilar central de las políticas educativas y proyectos educativos de los establecimientos educacionales y no se desarrolla ni trabaja explícitamente, manteniéndose invisible. Los investigadores recomiendan realizar un abordaje urgente ya que la confianza constituye un eje central de la calidad de vida y del vínculo humano pues es considerada como un componente constituyente del capital social.

\section{CONCLUSIONES}

En cuanto a la identificación del proceso vital de la política pública del instituto se encontró que posee estándares establecidos que enmarcan la prestación de los servicios en cada uno de los programas, dichos estándares se enfocan a proteger los derechos fundamentales de los niños a través de una atención integral establecida en la normatividad emanada por el estado Colombiano. En la norma se establece además la importancia de la participación activa del estado, la familia y la comunidad en la cual se desarrolla el menor. 
Por lo anteriormente descrito, el investigador concluye que es satisfactoria para los usuarios la atención brindada en los Centros de Desarrollo Infantil, en los cuales se cuenta con un equipo interdisciplinario que permite la atención integral del niño, donde se incluye el mejoramiento del proceso educativo por parte de los profesionales a los padres. Además, la infraestructura, condiciones de higiene y seguridad se encuentra adecuada para suplir todos los aspectos de atención directa, evaluación psicomotora y cognitiva del menor.

En cuanto a los hogares comunitarios de bienestar tradicional y FAMI, las condiciones de infraestructura y mobiliario no son adecuados ni seguros, no cuentan con las medidas de espacio, altura y tamaño de los sitios donde realizan sus actividades diarias los niños. Además, las condiciones de aseo, higiene y limpieza no son las mejores, se encuentran animales domésticos como perros, gatos, gallinas en los mismos espacios donde hacen sus actividades. La infraestructura donde se desarrollan los programas algunos no reúne condiciones mínimas. Las madres comunitarias en su mayoría, no son profesionales, requieren formación en las diferentes áreas del saber entre ellas ciencias de la educación, ciencias sociales, humanas y salud como se exige para el equipo interdisciplinario en los centro de desarrollo infantil, además se requiere de un número determinado de personas que garanticen la seguridad y protección del niño(a).

EI ICBF ha exigido la profesionalización de las madres comunitarias, las cuales han sido capacitadas a nivel de los programas por funcionarios de la entidad, pero aún existen debilidades en lo que respecta al maltrato infantil, convivencia pacífica, buen trato y violencia intrafamiliar, los cuales deben ser asumidos por profesionales competentes en el área.

Lo que respecta a los Hogares Comunitarios de Bienestar Familiar, donde se busca orientar a las madres de los menores y capacitarlas en temas de nutrición, cuidados, higiene para la familia, este estudio determinó que existen otros temas de interés para estas usuarias, los cuales representan una necesidad sentida, entre ellos son Planificación Familiar y Convivencia Familiar. Por tanto, se está educando en lo que se cree deben saber y no en lo que necesitan en realidad.

\section{REFERENCIAS}

1. Departamento Nacional de Planeación (DNP). CONPES Social 109, Política Pública Nacional de Primera Infancia "Colombia Por La Primera Infancia" [internet]. Citado en: noviembre 10, 2016. Disponible en:

http://www.mineducacion.gov.co/primerainfancia/1739/articles177828_archivo_pdf_conpes_109.pdf

2. Departamento Nacional de Planeación (DNP). CONPES Social 109, Política Pública Nacional de Primera Infancia "Colombia Por La Primera Infancia" [internet]. Citado en: noviembre 10, 2016. Disponible en:

http://www.mineducacion.gov.co/primerainfancia/1739/articles177828_archivo_pdf_conpes_109.pdf

3. Lerda, Acquatella y Gómez, El pensamiento complejo como alternativo al paradigma de Simplificación. Complejidad. Publicación trimestral Año 1. №3. 2003. pp. 2-9.

4. Cartilla No. 16. Higiene y Saneamiento Básico. Aldeas Infantiles SOS Colombia, Convenio Fortalecimiento Calidad Educación Inicial 2015 p 2. 
